\title{
Optimized Inter-Landmark Routing by Dtn-Flow Algorithm
}

\author{
Venothini. $\mathbf{N}^{1}$, Padmapriya. $\mathbf{V}^{2}$ \\ M.Tech, Department of Networking, Sri ManakulaVinayagar Engineering College, Puducherry, India ${ }^{1}$ \\ Assistant Professor, CSE, Sri ManakulaVinayagar Engineering College, Puducherry, India ${ }^{2}$
}

\begin{abstract}
This Paper focus on the well-organized routing of data in the middle of dissimilar areas in delay tolerant networks (DTN).In the current algorithm selects widely held places that nodes visit repeatedly as landmarks and splits the complete DTN area into subareas signified by landmarks. The number of node transfers between two landmarks is computed to represent the forwarding capability between them, relyupon which routing tables are created on each and every landmark to route the packet routing. However, there is more number of landmarks for a single path, throughput depends upon the traffic, the only static path is available, and no mobility nodes can be used. To overcome this problem, we suggest an Ad-hoc On Demand Distance Vector (AODV) routing technique in DTN flow algorithm to enhance node to node communication and for effective throughput is proposed. This technique can be used on all mobility nodes and efficient throughput can be obtained by the utilization of all nodes. The number of landmarks and the total cost is limited. Overall performance will be multiplied by increasing the $\mathrm{N}$ range of dynamic methods.
\end{abstract}

Keywords: Delay Tolerant Networks, inter-landmark, Ad-hoc on demand vector routing

\section{INTRODUCTION}

Delay tolerant networks (DTNs) are featured by irregular connection and numerous network partitions. Thus, DTN routing is typically accomplished during a carry-storeforward manner. We are predominantly involved in those that interchange data among or gather data from dissimilar areas because DTNs usually occur in areas without substructure networks and thereby are good modes to grasp data communication between these areas.

DTN routing algorithms abuse either historical encounter accounts (probabilistic routing), public network properties (social network routing), or past moving paths (locationbased routing) to deduce a node's probability of reaching a certain node or area, and forward packets to nodes with higher probability than current packet holder. The number of nodes with high probability of visiting the destination usually is limited, by only relying on such nodes, previous routing algorithms fail to fully utilize all node movements, leading to degraded overall throughput.

The design principles of DTN architecture can be summarized as follows:

- Variable length messages will exist as the communication abstraction to facilitate the ability of the network for scheduling or path selection decisions.

- A naming syntax which supports a wide a range of naming and addressing conventions is applied to enhance interoperability.

- Storage within the network is taken to support store and forward operation over multiple paths and potentially long time scales.

- Security mechanisms are provided to protect the infrastructure from unauthorized users by discarding traffic as quickly as possible.

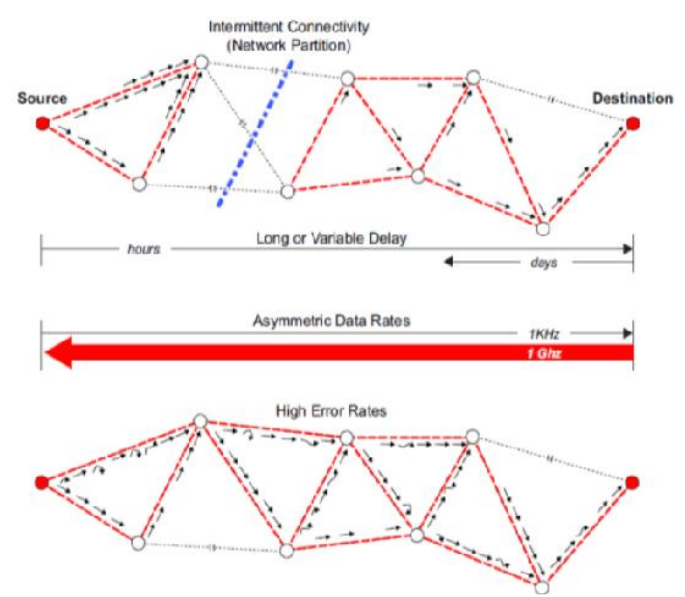

Fig.1. DTN Architecture Principles

\section{RELATED WORK}

Anders Lindgreny, Avri Doria, Olov Schelen(2003),this paper addresses the matter of routing in intermittently connected networks. In such networks there's no guarantee that a totally connected path between sender and destination exists at any time, rendering ancient routing protocols unable to deliver messages between hosts. So, they proposed PROPHET [1], a probabilistic routing protocol for intermittently connected networks and compare it to the earlier given Epidemic Routing protocol through simulations. They tend to show that PROPHET is ready to deliver additional messages than Epidemic Routing with a lower communication overhead.

Pan Hui, Jon Crowcroft, EikoYoneki (2008), propose a social based mostly forwarding formula[2], BUBBLE that is shown by trial and error to boost the forwarding potency considerably compared to oblivious forwarding schemes 
and to PROPHET formula. They tend to additionally show, however this formula is enforced during a $A$. distributed manner that demonstrates that it's applicable within the decentralized surroundings of PSNs.

Quan Yuan, IonutCardei and Jie Wu (2009),proposes a Predict and Relay (PER)[3], an efficient routing algorithm for DTNs, wherever nodes verify the probability distribution of future contact times and select a correct next hop so as to boost the end-to-end delivery probability. The algorithm is predicated on 2 observations: one is that nodes sometimes move around a collection of well visited landmark points rather than moving randomly; the opposite is that node quality behaviour is semideterministic and will be expected once there's sufficient quality history information. Specifically, this method improves the delivery ratio and additionally reduces the delivery latency compared to traditional DTN routing schemes.

M. Lin, W.-J. Hsu, and Z. Q. Lee (2012), this research investigates the scaling effects on predictability [4]. Given specific spatial-temporal scales, recorded trajectories area unit encoded into long strings of distinct locations, and several other information-theoretic measures of predictability area unit derived. Somewhat amazingly, high predictability is still gift at terribly high spatial/temporal resolutions. Moreover, the predictability is freelance of the mobility area covered.

This implies extremely regular quality behaviours. Moreover, by varied the scales over a wide range, invariability is observed that suggests that bound tradeoffs between the predicting accuracy and spatial-temporal resolutions unavoidable ineluctable. As several applications in ubiquitous computing concern quality, these findings ought to have direct implications.

K. Chen and H. Shen (2013), they propose DTN-FLOW [5], an efficient routing algorithm to transfer data among landmarks with high throughput in DTNs. DTN-FLOW splits the entire DTN area into subareas with different landmarks and uses node transits between landmarks to forward packets one landmark by one landmark to reach their destinations.It fully utilizes all node movements to route packets along landmark-based paths to their destinations.

The main disadvantages are, there is more number of landmarks for a single path, throughput depends upon the traffic, only static path is available, no mobility nodes can be used. The major disadvantages of DTN networks are there is more number of landmarks for a single path, throughput depends upon the traffic, only static path is available, no mobility nodes can be used.

To overcome this problem an AODV (Ad-hoc on demand distance vector) routing technique in DTN flow algorithm to enhance node to node communication and for effective throughput is proposed.

\section{SYSTEM DESIGN}

LANDMARK SELECTION AND SUB AREA

The landmark selection decides the places to install landmarks. Subarea partition assigns each landmark a subarea. Both landmark selection and subarea division are conducted by the network supervisor or planner who hopes to utilize the DTN for a certain application.

1) Landmark Selection: As above-mentioned, we select popular places that are often visited by mobile nodes as landmarks. To identify popular places, a simple way is to gather node visiting history and take the top most often visited places as popular places. Popular places in DTNs with social network structures can also be predetermined based on node mobility pattern. For example, in the DART network, we can easily find popular buildings that students visit frequently: library, department buildings, and dorms. In DTNs in rural areas, villages are naturally popular places. In the DTNs using animals as mobile nodes for environment monitoring in mountain areas, places with water/food are frequently visited. The resulted popular places form a candidate landmark list. There may be several popular places in a small area. Thus, not every popular place needs to be a landmark. Then, for every two candidate landmarks with distance less than meters, the one with less visit occurrence is removed from the candidate list. Finally, the distance between every two candidate landmarks is larger than sub area meters.

2) Subarea Division: With the landmarks, we split the entire network into subareas. Since the subarea division only serves the purpose of routing among landmarks, we do not need a method to accurately define the size of each subarea. Therefore, we follow the rules below to generate subareas.

- Each subarea contains only one landmark.

- The area between two landmarks is evenly split to the two subareas containing the two landmarks.

- There is no overlap among subareas.

\section{B. NODE TRANSIT PREDICTION}

Since DTN-FLOW relies on node passage for packet forwarding, accurate expectation of node transit is a key component. DTN-FLOW forecasts each node's next transit by preserving a landmark visiting history table on each node. Each node predicts its transits based on its preceding landmark visiting records using the order Markov predictor.

\section{ROUTING TABLE CONSTRUCTION}

In DTN-FLOW, each landmark dynamically processes the bandwidths of its transit links to each neighbour landmark. The bandwidth of a transit link represents the predictable delay of forwarding data through it.

Based on the estimated delay, each landmark uses the distance-vector method [6] to build a routing table indicating the next-hop landmark for each destination landmark. 
Each landmark intermittently transfers its routing table to its neighbour landmarks for routing table update. This step is recognized through mobile nodes, i.e., a landmark; say $\mathrm{L}_{\mathrm{i}}$, chooses its node with the highest predicted probability of visiting $L_{j}$ to forward its routing table to $L_{j}$.

\section{PACKET FORWARDING ALGORITHM}

During the packet dispatching, a landmark refers to its routing table to select the next-hop landmark and forwards the packet to the mobile node that has the highest expected probability to transit to the next-hop landmark. However, node transit prediction may not always be accurate, which means a node may fail to carry a packet to the landmark specified in the routing table. Also, there may be nodes that are moving to the packet's destination node directly, which can be utilized to enrich the routing performance. We first introduce our methods to handle the two issues and then review the routing algorithm.

1)Handling Prediction Inaccuracy: To handle the wrong transit prediction, DTN-FLOW [5] follows the principle that each forwardshould cut back the routing latency. Thus, when a node moves from $\mathrm{L}_{\mathrm{i}}$ to a landmark $\mathrm{L}_{\mathrm{k}}$ apart from the expected one $\mathrm{L}_{\mathrm{j}}$, the node checks whether the new landmark still reduces the expected delay to the destination $\mathrm{L}_{\mathrm{d}}$, that is, whether $\mathrm{D}\left(\mathrm{L}_{\mathrm{k}}, \mathrm{L}_{\mathrm{d}}\right)<\mathrm{D}\left(\mathrm{L}_{\mathrm{i}}, \mathrm{L}_{\mathrm{d}}\right)$. If yes, the node still forwards the packet to landmark for more forwarding. Otherwise, the node holds the packet, looking forward to next landmark that has shorter delay to the destination. This design aims to make sure that every transit, though might not be optimum because of node transit prediction inaccuracy, can always improve the change of successful delivery.

2) Exploiting Direct Delivery Opportunities:Since nodes move opportunistically in a DTN, it is possible that a landmark can discover nodes that are predicted to visit the destination landmarks of some packets. Therefore, when a landmark receives a packet, it first checks whether any connected nodes are predicted to transit to its destination landmark. If yes, the packet is forwarded to the node directly. In case the node fails to forward the packet to its destination landmark, the node uses the scheme described in Section IV-D.1 to decide whether to forward the packet to the new landmark.

3) Routing Algorithm: We present the steps of the routing algorithm as follows.

1) 1)If the node generates a packet for a section, it just forwards the packet to the landmark when it meets first.

2) At the point when a landmark, say $\mathrm{L}_{\mathrm{i}}$, creates or gets a bundle, it first checks whether any nodes are anticipated to move to the destination historic point of the bundle. On the off chance that yes, the bundle is sent to the hub with the most anticipated probability along with the expected overall delay, which is utilized by the transportation node to figure out if to forward the bundle to an experienced historic point other than the anticipated one.
3) Overall $\mathrm{L}_{\mathrm{i}}$, checks its steering table to discover the following jump historic point for the bundle and supplements the landmark ID and the normal general postponement into the bundle.

4) Li then checks all associated nodes and advances the bundle to the node that has accessible memory and has the most elevated anticipated probability to travel to the following bounce landmark demonstrated by the directing table.

5) At the point when a node moves to the region of a landmark, say $\mathrm{Lj}$, it Forwards $\mathrm{Lj}$ all bundles that target $\mathrm{Lj}$ or have less overall delay from $\mathrm{Lj}$ to the destination than $\mathrm{L}_{\mathrm{i}}$. After this, it predicts its next travel in light of the order-Markov indicator and illuminates this to $\mathrm{Lj}$.

\section{CONCLUSION}

In this paper, the data is carried between landmarks and high throughput is sustained in DTN. The entire DTN area is divided into subareas each having different landmarks and uses node transits between landmarks to reach their destinations using the DTN-FLOW Algorithm. The number of node transfers between two landmarks is computed to represent the forwarding capability between them, depend on which routing tables are created on each landmark to guide packet routing. An Ad-hoc on demand distance vector routing technique in DTN-FLOW algorithm to combine node to node communication to enhance packet routing efficiency. In future, this work is further extended by using any other routing technique to achieve effective throughput.

\section{REFERENCES}

11 A.Lindgren, A. Doria, and O. Schelén, "Probabilistic routing in intermittently connected networks," Mobile Comput. Commun. Rev., vol. 7,no. 3, pp. 19-20, 2003.

[2] P. Hui, J. Crowcroft, and E. Yoneki, "Bubble rap: Social-based forwarding in delay tolerant networks," in Proc. ACM MobiHoc, 2008,pp. 241-250.

[3] Q. Yuan, I. Cardei, and J. Wu, "Predict and relay: An efficient routing in disruption-tolerant networks," in Proc. ACM MobiHoc, 2009, pp.95-104.

[4] M. Lin, W.-J. Hsu, and Z. Q. Lee, "Predictability of individuals mobility with high-resolution positioning data," in Proc. UbiComp, 2012,pp. 381-390.

[5] K. Chen and H. Shen, "DTN-FLOW: Inter-landmark data flow for high-throughput routing in DTNs," in Proc. IEEE IPDPS, 2013, pp.726-737.

[6] C. Hedrick, "RFC1058-Routing information protocol," 1988 